\title{
Paving the way for E-vita open NEO hybrid prosthesis implantation for complex aortic arch disease in Asia-Pacific
}

\author{
Heinz Jakob ${ }^{1}$, Jacky YK Ho ${ }^{2}$, Hung Leung Randolph Wong ${ }^{2}$, Mohammed Idhrees ${ }^{3}$, Bashi \\ Velayudhan $^{3}$, George Matalanis ${ }^{4}$, Daniel- Sebastian Dohle ${ }^{5}$, Klaus Görlinger ${ }^{6}$, and \\ Mohamad Bashir ${ }^{7}$ \\ ${ }^{1}$ Diagnosticum Mülheim \\ ${ }^{2}$ Prince of Wales Hospital \\ ${ }^{3} \mathrm{SRM}$ Institutes for Medical Science Vadapalani \\ ${ }^{4}$ Austin Hospital \\ ${ }^{5}$ Johannes Gutenberg Universitat Mainz \\ ${ }^{6}$ Tem Innovations GmbH \\ ${ }^{7}$ Royal Blackburn Teaching Hospital
}

June 10, 2021

\begin{abstract}
We report first in man implantations of the newly designed Evita-open-NEO hybrid prosthesis (HP) for complex aortic arch disease from 3 different countries in Asia-Pacific including suggestions on how to proceed with optimized perioperative coagulation.
\end{abstract}

\section{Introduction}

Frozen elephant trunk (FET) has evolved over the years in technology and techniques. The new generation E-vita-Open-NEO (JOTEC CryoLife), is now available in 3 variations. We report first in man implantations from 3 different countries in Asia-Pacific with a note on perioperative coagulation management

The article does not represent a clinical trial or an international multicentre study. It is a direct case reporting reporting of experience and outcomes, henceforth, IRB approval was note required. Further informed conset from each of the three patients has has been individually obtained. Moreover, institutional approval for use of Evita-open-NEO (CE market approval, March 2020) has been approved locally by governance boards at every reporting centres.

\section{Case Reports (Table 1)}

Hong Kong: A 60 year, female diagnosed with a Type III Aortic dissection (AD) and arch aneurysm who underwent total arch replacement (TAR) with FET using a branched Evita-open-NEO. Patient details are provided in table 1. There was no significant oozing encountered after protamine administration. Transfusion was according to thromboelastometry guided coagulation management (TGCM) with no further postoperative transfusion was required. She was neurologically intact and discharged on day 12 .

Chennai: A 54-year male was diagnosed to have ascending and aortic arch aneurysm $(5.2 \mathrm{~cm}$ at zone 1$)$ with Type B AD. Patient details are provided in table 1. After protamine administration there was continuous oozing from the polyester segment of the prosthesis. Oozing was controlled by covering the prosthesis with Surgicel-Fibrillar (Johnson \& Johnson) and pressurized packing with gauze for few minutes. Blood 
transfusion and postoperative drain as in table 1 . He was discharged on the $10^{\text {th }}$ postoperative day and follow-up CT aortogram was done (Figure 1A/B).

Melbourne: An 81 year old male underwent TAR with FET technique using trifurcate 30/40/180mm Evita-Open-NEO HP. In addition he received a Bioprosthetic Bentall's procedure and coronary artery bypass graft (Left internal mammary artery and Left Radial). Patient details are provided in table 1. Blood and products were transfused according to TGCM. No excessive oozing from the fabric of the graft or any of its links has been observed, and drain losses were minimal. Postoperative details elaborated in Table 1. Palliation was instituted as per family wishes (Figure 1C/D).

\section{DISCUSSION}

HP for FET is constantly evolving through the years ever since the first device - 'Essen I prosthesis' (2005) (1). Given the success of Evita-open-plus across the globe, researchers have developed the next generation HP: Evita-open-NEO. The new generation prosthesis is available in three variations, making it suitable to be implanted in zone 0 to 3 , and the supraaortic-arch-vessels can be reimplanted individually or en-bloc. This provides a wide range of applicablity for the surgeons emcomprising different pathological situations. The low profile delivery system and a shapeable shaft, is atraumatic and helps in precise deployment (2).

Evita-open-NEO prostheses are not impregnated with gelatin or collagen, hence some discrete oozing through pores of the tightly woven polyester during heparinization can be expected but easily controlled by sump suction.

This oozing is self-limiting and stops once the protamine is administered after weaning of cardiopulmonary bypass ( $\mathrm{CPB})$ supported by packing the mediastinum with sponges and towels for 10-15 minutes. Blood collected in mediastinum can be efficiently managed with cell saver. In patients who undergo FET under prolonged deep hypothermic circulatory arrest, coagulopathy has to be considered. In that case, graft oozing through the pores may appear excessive but can be quickly fixed by TGCM during and after CPB (Video 1).

Thromboelastometry provides important information about hemostasis, particularly in regard to fibrin polymerization and platelet function, and can already be initiated at crossclamp release. Accordingly, cryoprecipitate can timely be prepared if fibrinogen concentrate is not available. Adequate fibrin and platelet contribution to the clot firmness are essential for the formation of an internal fibrin layer to seal the prosthesis (3). In contrast, low fibrinogen levels $(<2-4 \mathrm{~g} / \mathrm{L})$ have been shown to be an independent risk-factor for severe bleeding, 24-hour mortality, in-hospital mortality, and neurological complications in patients with acute $\mathrm{AD}(3-6)$.

The algorithm of TGCM for major aortic surgery is by using a trigger of EXTEM A $5<40 \mathrm{~mm}$ and FIBTEM A5 $<12 \mathrm{~mm}$, and a target of EXTEM A5 [?] $40 \mathrm{~mm}$ and FIBTEM A5 [?]15 $\mathrm{mm}$ for fibrinogen substitution (3, 7). Notably, this can only be achieved by administering fibrinogen concentrate $(20 \mathrm{~g} / \mathrm{l})$ or cryoprecipitate (8-16 g/l) but not by FFP containing only $2 \mathrm{~g}$ fibrinogen/l) (8). Some patients require platelet transfusion and/or prothrombin complex concentrate administration in addition.

In contrast, Czerny and colleagues recently reported devastating oozing and severe blood loss in 3 patients after implantation of E-vita open NEO (9). Those patients, characterized by continuous acetylic acid monotherapy preoperatively, obviously mandated differentiated coagulation management during and after $\mathrm{CPB}$. Here, FFP transfusion with its low fibrinogen concentration is certainly not appropriate and may cause pulmonary complications, right ventricular failure, and increased RBC transfusion $(10,12)$.

\section{Conclusion}

In combination with TGCM, the new Evita-open-NEO HP can easily and safely be implanted with minimal blood loss.

Conflict of Interest: Heinz Jakob is consultant to JOTEC/CryoLife, former inventor and recipient of royalties until 2019; Klaus Görlinger is the medical director of Tem Innovations GmbH, Munich, Germany 


\section{Reference}

1. Jakob H, Tsagakis K, Leyh R, et al. Development of an integrated stent graft-dacron prosthesis for intended one-stage repair in complex thoracic aortic disease. Herz. 2005 Dec;30(8):766-8. doi: 10.1007/s00059-005-2765-7

2. Ho JYK, Bashir M, Jakob $\mathrm{H}$ and RH Wong, (2021) Management of left subclavian artery in total arch replacement and frozen elephant trunk, JTCVS Techniques, article-in-press, https://doi.org/10.1016/j.xjtc.2021.03.020

3. Hanke AA, Herold U, Dirkmann D, et al. Thromboelastometry Based Early Goal-Directed Coagulation Management Reduces Blood Transfusion Requirements, Adverse Events, and Costs in Acute Type A Aortic Dissection: A Pilot Study. Transfus Med Hemother. 2012 Apr;39(2):121-128. doi: 10.1159/000337723.

4. Guan X, Gong M, Wang X, et al. Low preoperative fibrinogen level is risk factor for neurological complications in acute aortic dissection. Medicine (Baltimore). 2018 May;97(21):e10830. doi: 10.1097/MD.0000000000010830.

5. Liu J, Sun LL, Wang J, Ji G. The relationship between fibrinogen and in-hospital mortality in patients with type A acute aortic dissection. Am J Emerg Med. 2018 May;36(5):741-744. doi: 10.1016/j.ajem.2017.10.00

6. Monaco F, Nardelli P, Denaro G, et al. First experience with a ROTEM-enhanced transfusion algorithm in patients undergoing aortic arch replacement with frozen elephant trunk technique. A theranostic approach to patient blood management. J Clin Anesth. 2020 Nov;66:109910. doi: 10.1016/j.jclinane.2020.109910.

7. Görlinger K, Pérez-Ferrer A, Dirkmann D, et al. The role of evidence-based algorithms for rotational thromboelastometry-guided bleeding management. Korean J Anesthesiol. 2019 Aug;72(4):297-322. doi: 10.4097/kja.19169.

8. Görlinger K, Shore-Lesserson L, Dirkmann D, et al. Management of hemorrhage in cardiothoracic surgery. J Cardiothorac Vasc Anesth. 2013 Aug;27(4 Suppl):S20-34. doi: 10.1053/j.jvca.2013.05.014..

9. Czerny M, Beyersdorf F, Murana G, Pacini D. Excessive oozing through the fabric of the branched Cryolife-Jotec Evita Open NEO hybrid prosthesis. Eur J Cardiothorac Surg. 2021 Mar 17:ezab058. doi: 10.1093/ejcts/ezab058.

10. Monaco F, Barucco G, Nardelli P, et al. Editor's Choice - A Rotational Thromboelastometry Driven Transfusion Strategy Reduces Allogenic Blood Transfusion During Open Thoraco-abdominal Aortic Aneurysm Repair: A Propensity Score Matched Study. Eur J Vasc Endovasc Surg. 2019 Jul;58(1):13-22. doi: $10.1016 /$ j.ejvs.2019.02.009

11. Narick C, Triulzi DJ, Yazer MH. Transfusion-associated circulatory overload after plasma transfusion. Transfusion. 2012 Jan;52(1):160-5. doi: 10.1111/j.1537-2995.2011.03247.x.

12. Desborough M, Sandu R, Brunskill SJ, et al. Fresh frozen plasma for cardiovascular surgery. Cochrane Database Syst Rev. 2015 Jul 14;(7):CD007614. doi: 10.1002/14651858.CD007614.

\section{Legend}

Figure 1: (A)Preoperative CT aortogram showing the ascending \& aortic arch aneurysm. (B)Postoperative image depicting a well expanded true lumen and thrombosed false lumen without distal stent induced new entry (DSINE). (C)Preoperative CT scan demonstrating a $9 \mathrm{~cm}$ distal arch aneurysm and a heavily calcified aortic valve. (D)Postoperative CT scan demonstrating the Bentall root replacement and full exclusion of distal arch aneurysm without DSINE.

Figure 2: Algorithm TGCM in major aortic surgery [modified from (7)]

Video 1: Demonstrating the effect of TCGM (A)Without TGCM: prolonged oozing (B)With TGCM: hemostasis achieved within 30 minutes of protamine administration. 


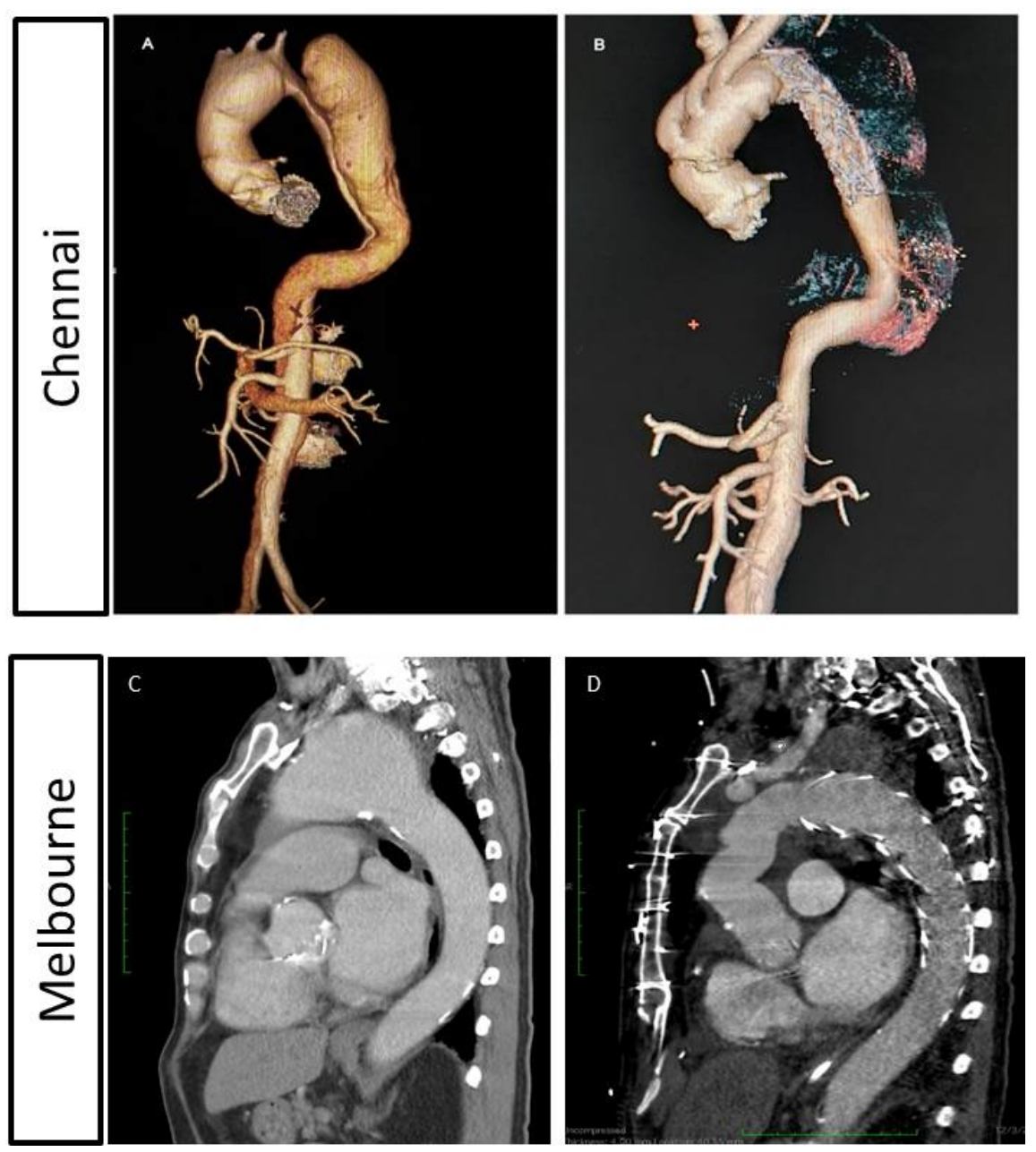




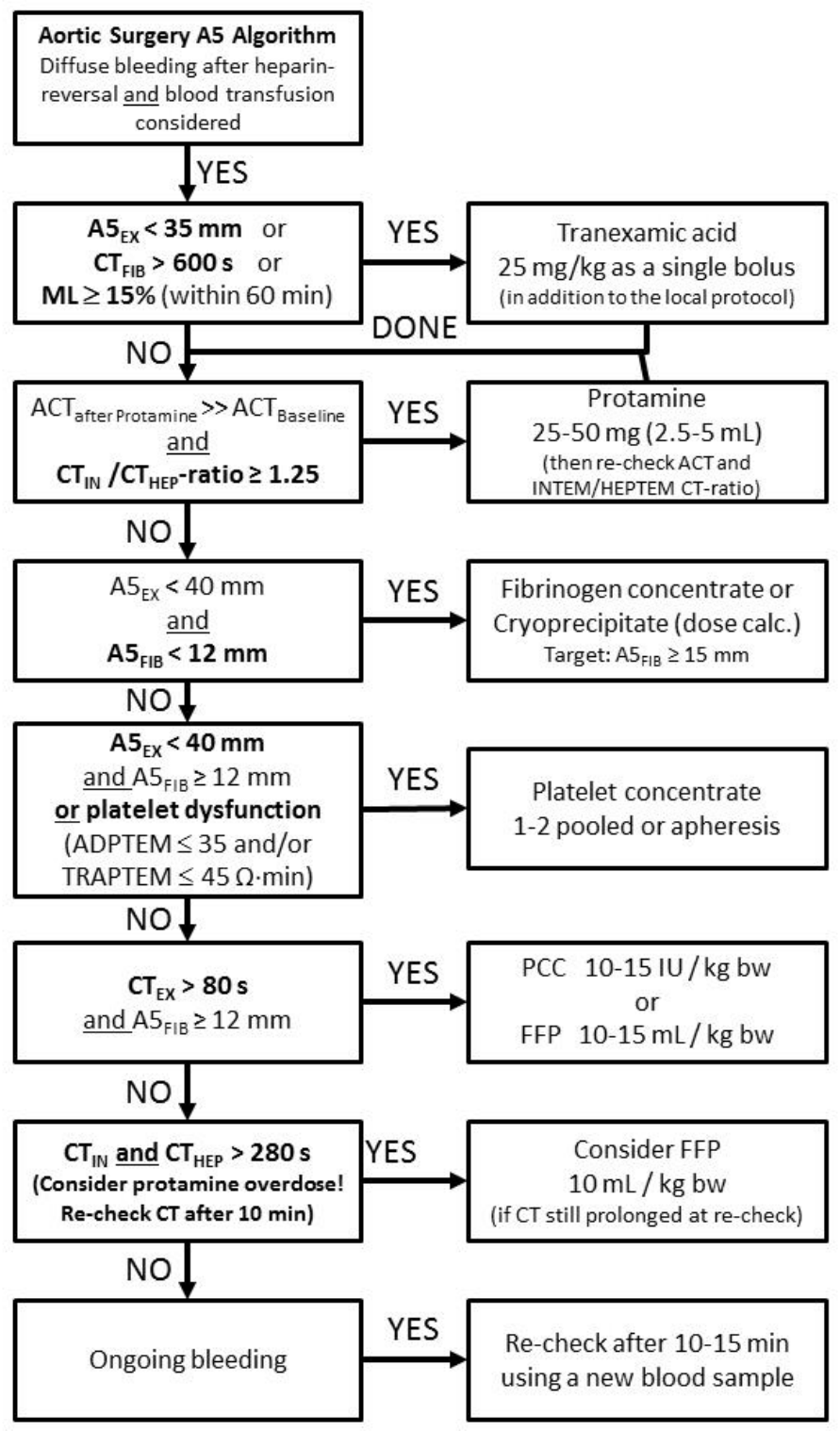

